\title{
Statins and the COVID-19 main protease: in silico evidence on direct interaction
}

\author{
Željko Reiner ${ }^{1}$, Mahdi Hatamipour ${ }^{2,3}$, Maciej Banach ${ }^{4,5}$, Matteo Pirro ${ }^{6}$, Khalid Al-Rasadi ${ }^{7}$, \\ Tannaz Jamialahmadi ${ }^{8,9}$, Dina Radenkovic ${ }^{10}$, Fabrizio Montecucco ${ }^{11,12}$, Amirhossein Sahebkar ${ }^{13,14,15}$
}

\author{
${ }^{1}$ Department of Internal Medicine, University Hospital Centre Zagreb, Zagreb, Croatia \\ ${ }^{2}$ Nanotechnology Research Centre, Pharmaceutical Technology Institute, \\ Mashhad University of Medical Sciences, Mashhad, Iran \\ ${ }^{3}$ Department of Medicinal Chemistry, School of Pharmacy, Mashhad University \\ of Medical Sciences, Mashhad, Iran \\ ${ }^{4}$ Department of Hypertension, WAM University Hospital in Lodz, Medical University \\ of Lodz, Lodz, Poland \\ 5Polish Mother's Memorial Hospital Research Institute (PMMHRI), Lodz, Poland \\ ${ }^{6}$ Unit of Internal Medicine, Angiology, and Arteriosclerosis Diseases, \\ Department of Medicine, University of Perugia, Perugia, Italy \\ 'Department of Clinical Biochemistry, Sultan Qaboos University Hospital, Muscat, Oman \\ ${ }^{8}$ Biotechnology Research Centre, Pharmaceutical Technology Institute, \\ Mashhad University of Medical Sciences, Mashhad, Iran \\ ${ }^{9}$ Department of Nutrition, Faculty of Medicine, Mashhad University of Medical \\ Sciences, Mashhad, Iran \\ ${ }^{10}$ Guy's \& St Thomas' NHS Foundation Trust, London SE1 7EH, UK \\ ${ }^{11}$ First Clinic of Internal Medicine, Department of Internal Medicine and Centre \\ of Excellence for Biomedical Research (CEBR), University of Genoa, Genoa, Italy \\ ${ }^{12}$ IRCCS Ospedale Policlinico San Martino Genoa - Italian Cardiovascular Network, \\ Genoa, Italy \\ ${ }^{13}$ Halal Research Center of IRI, FDA, Tehran, Iran \\ ${ }^{14}$ Biotechnology Research Centre, Pharmaceutical Technology Institute, \\ Mashhad University of Medical Sciences, Mashhad, Iran \\ ${ }^{15}$ Neurogenic Inflammation Research Centre, Mashhad University of Medical Sciences, \\ Mashhad, Iran
}

Submitted: 12 April 2020

Accepted: 20 April 2020

Arch Med Sci 2020; 16 (3): 490-496

DOI: https://doi.org/10.5114/aoms.2020.94655

Copyright @ 2020 Termedia \& Banach

\section{Abstract}

Introduction: No proven drug and no immunisation are yet available for COVID-19 disease. The SARS-CoV-2 main protease (Mpro), a key coronavirus enzyme, which is a potential drug target, has been successfully crystallised. There is evidence suggesting that statins exert anti-viral activity and may block the infectivity of enveloped viruses. The aim of this study was to as sess whether statins are potential COVID-19 Mpro inhibitors, using a molecular docking study.

Material and methods: Molecular docking was performed using AutoDock/ Vina, a computational docking program. SARS-CoV-2 Mpro was docked with all statins, while antiviral and antiretroviral drugs - favipiravir, nelfinavir, and lopinavir - were used as standards for comparison.

Results: The binding energies obtained from the docking of 6LU7 with native ligand favipiravir, nelfinavir, lopinavir, simvastatin, rosuvastatin, pravastatin, pitavastatin, lovastatin, fluvastatin, and atorvastatin were $-6.8,-5.8$, $-7.9,-7.9,-7.0,-7.7,-6.6,-8.2,-7.4,-7.7$, and $-6.8 \mathrm{kcal} / \mathrm{mol}$, respectively. The number of hydrogen bonds between statins and amino acid residues of Mpro were 7, 4, and 3 for rosuvastatin, pravastatin, and atorvastatin, respectively, while other statins had two hydrogen bonds.

Conclusions: These results indicate, based upon the binding energy of pitavastatin, rosuvastatin, lovastatin, and fluvastatin, that statins could be

\author{
Corresponding authors: \\ Prof. Maciej Banach \\ Department of Hypertension \\ WAM University Hospital \\ Medical University of Lodz \\ 113 Zeromskiego St \\ 90-549 Lodz, Poland \\ Phone: +48 426393771 \\ Fax: +48 4263937 71 \\ E-mail: maciejbanach77@ \\ gmail.com
}

Prof. Amirhossein Sahebkar Biotechnology Research Centre Pharmaceutical Technology Institute Mashhad University of Medical Sciences Mashhad 9177948564, Iran Phone: +98 5138002299 Fax: +98 5138002287 E-mail: sahebkara@mums.ac.ir; amir_saheb2000@yahoo.com 
efficient SARS-CoV-2 Mpro inhibitors. This is supported by the fact that the effects of some statins, especially pitavastatin, have a binding energy that is even greater than that of protease or polymerase inhibitors. However, further research is necessary to investigate their potential use as drugs for COVID-19.

Key words: COVID-2019, main protease, statins, 6LU7, docking.

\section{Introduction}

A new strain of coronavirus called SARS-CoV-2 (Severe Acute Respiratory Syndrome CoronaVirus 2) causing an infectious disease called COVID-19 (COronaVIrus Disease 2019) was first identified in Wuhan, the capital of China's Hubei province, in December 2019 as the aetiological agent responsible for the viral pneumonia outbreak [1]. The World Health Organisation (WHO) declared the outbreak in China on 30 January 2020 to be a Public Health Emergency of International Concern (PHEIC) [2]. Due to the rapid increase in the numbers of patients outside China and the growing number of countries that were involved, on 12 March 2020 the WHO stated that COVID-19 was characterised as a pandemic. To date, more than 2.3 million people have contracted this disease, and more than 170,000 have died because of it. No proven specific drugs and no immunisation are available so far, while therapeutic options are very limited [3, 4]. In fact, only preventive and supportive therapies directed to prevent complications of the disease and organ damage are available. Although antimalarial drugs chloroquine and hydroxychloroquine are used in some countries for the treatment of this disease, at present there is not sufficient evidence to determine whether they are effective treatments for COVID-19 [5].

However, the antagonisms with other drugs and serious adverse effects (SAEs) of these drugs are also well known. Lopinavir/ritonavir, protease inhibitors that have been widely used for the treatment of human immunodeficiency virus-1 (HIV-1) infection, as a combination and in combination with azithromycin, have been used on a small scale for treatment of COVID-19 patients with some success [6]. Favipiravir, which electively and potently inhibits the RNA-dependent RNA polymerase, has also been used to treat some patients with this disease [7], as have nucleoside analogues, neuraminidase inhibitors, remdesivir, some peptides (EK1), umifenovir, and RNA synthesis inhibitors (such as TDF and 3TC), but for all these drugs the results of studies on more patients are awaited because their useful effects on COVID-19 are still anecdotal.

Statins are conventionally known as effective cholesterol-lowering drugs. Moreover, these drugs possess other beneficial effects independent of their cholesterol-lowering effect, including anti-in- flammatory, anti-thrombotic, and immunomodulatory functions [8-15]. Furthermore, statin treatment can interfere with viral infectivity through inhibition of glycoprotein processing. Disruption of protease activity and decreased ratios of the mature glycoproteins to precursor form are possible mechanisms of the antiviral effect of statins. Disruption of the viral protease activity is an important therapeutic goal [16].

The main protease (Mpro) of a virus, in this case CoV, plays an important role in proteolytic maturation [17]. Consequently, it has been examined as a potential protein target to prevent infection expansion by inhibiting the cleavage of the viral polyprotein [18]. This might explain why statins could be useful in the treatment of COVID-19. The aim of this study was to assess whether statins might be potential COVID-19 Mpro inhibitors. Namely, most recently the SARS-CoV-2 main protease (Mpro), a key coronavirus enzyme, which is a potential drug target, has been successfully crystallised $[19,20]$. Therefore, the idea was to investigate whether statins could be useful in the treatment of COVID-19 by directly affecting the virus particle.

\section{Material and methods}

\section{Ligand preparation}

All the molecules were constructed with the ChemDraw v2015 program, and these geometries energies were minimised by using the MOE v2015, with Force Field type AMBER 10. The created .pdb file was submitted to ADT to set number of torsion and for .pdbqt file construction.

\section{Protein preparation}

Three-dimensional coordinates of COVID-19 Mpro (PDB ID: 6LU7) were obtained in .pdb format from the Protein Data Bank (https:/www. rcsb.org/). The .pdb file was entered into AutodockTools (ADT ver.1.5.6) for preparation of a .pdbqt file and grid box creation. Water molecules and other atoms were excluded, and ADT measured the Gasteiger charges for protein atoms; AutoGrid was used with a grid box to create the grid map. The size of grid was determined at $60 \times 60$ $\times 60$ xyz points with a grid spacing of $0.375 \AA$, and a grid centre at dimensions ( $x, y$, and $z$, respectively): $-9.732,11.403$, and 68.925 was designated. 
Table I. Interaction of tested ligands with SARS-CoV-2 main protease

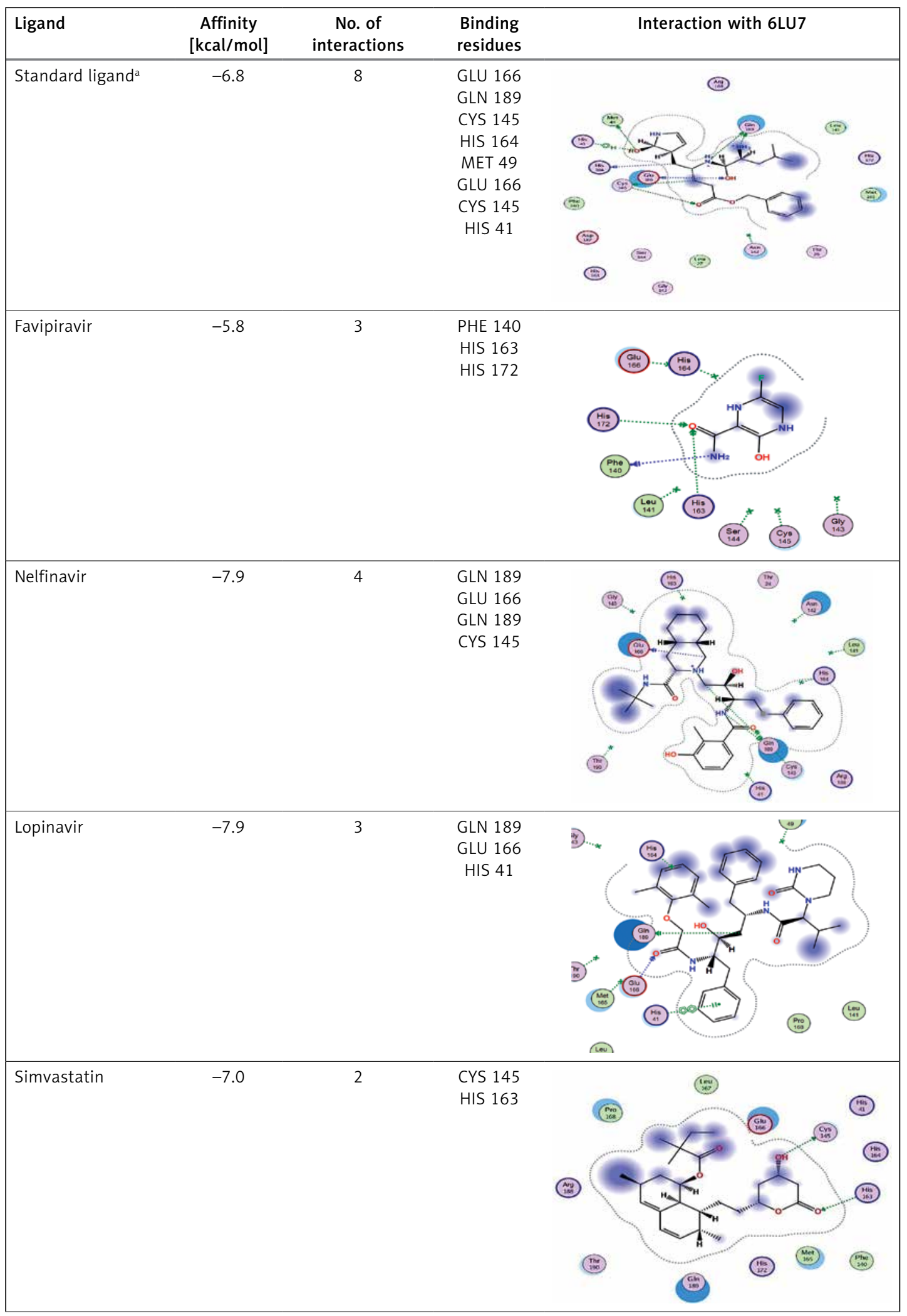


Table I. Cont.

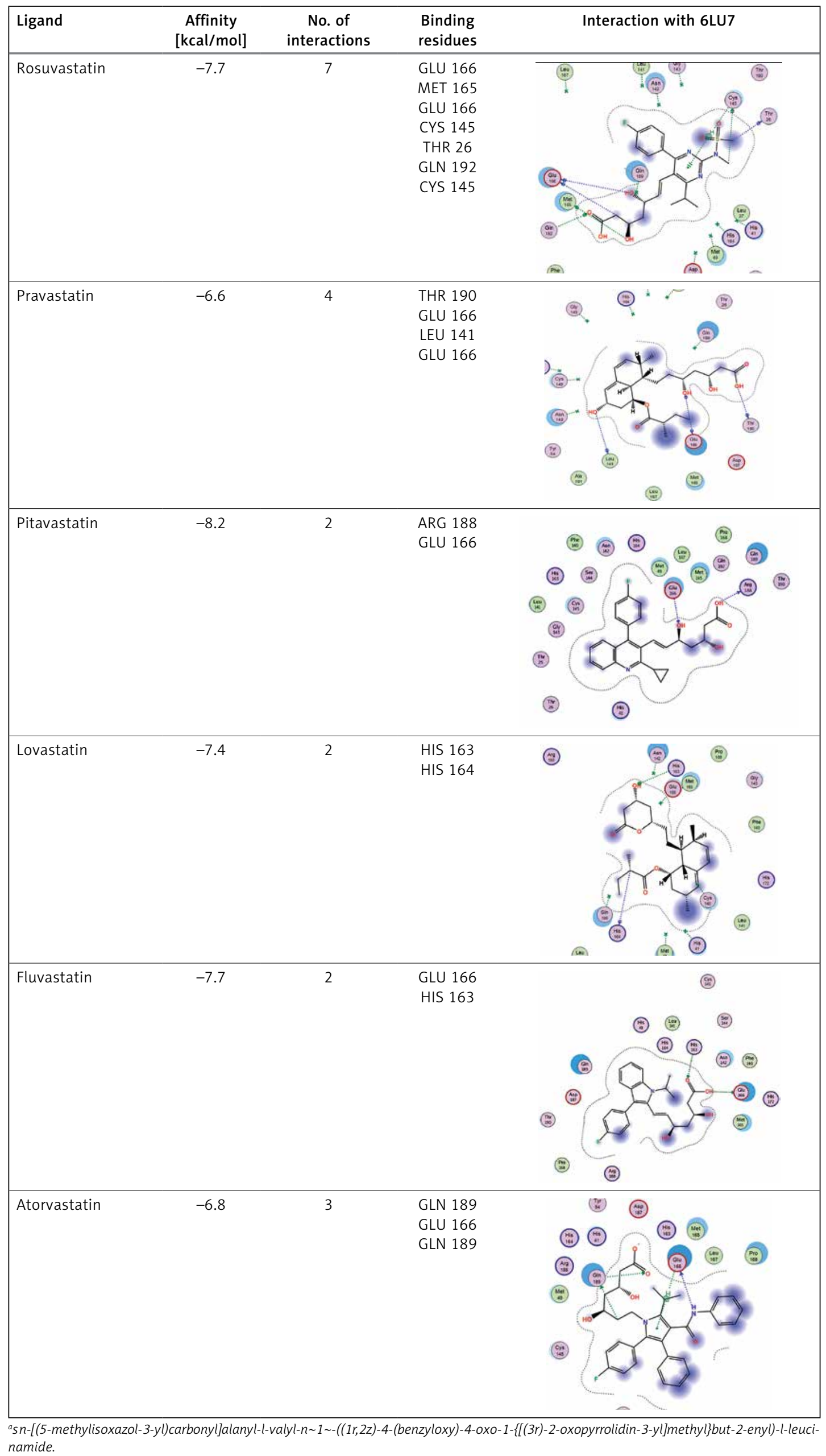



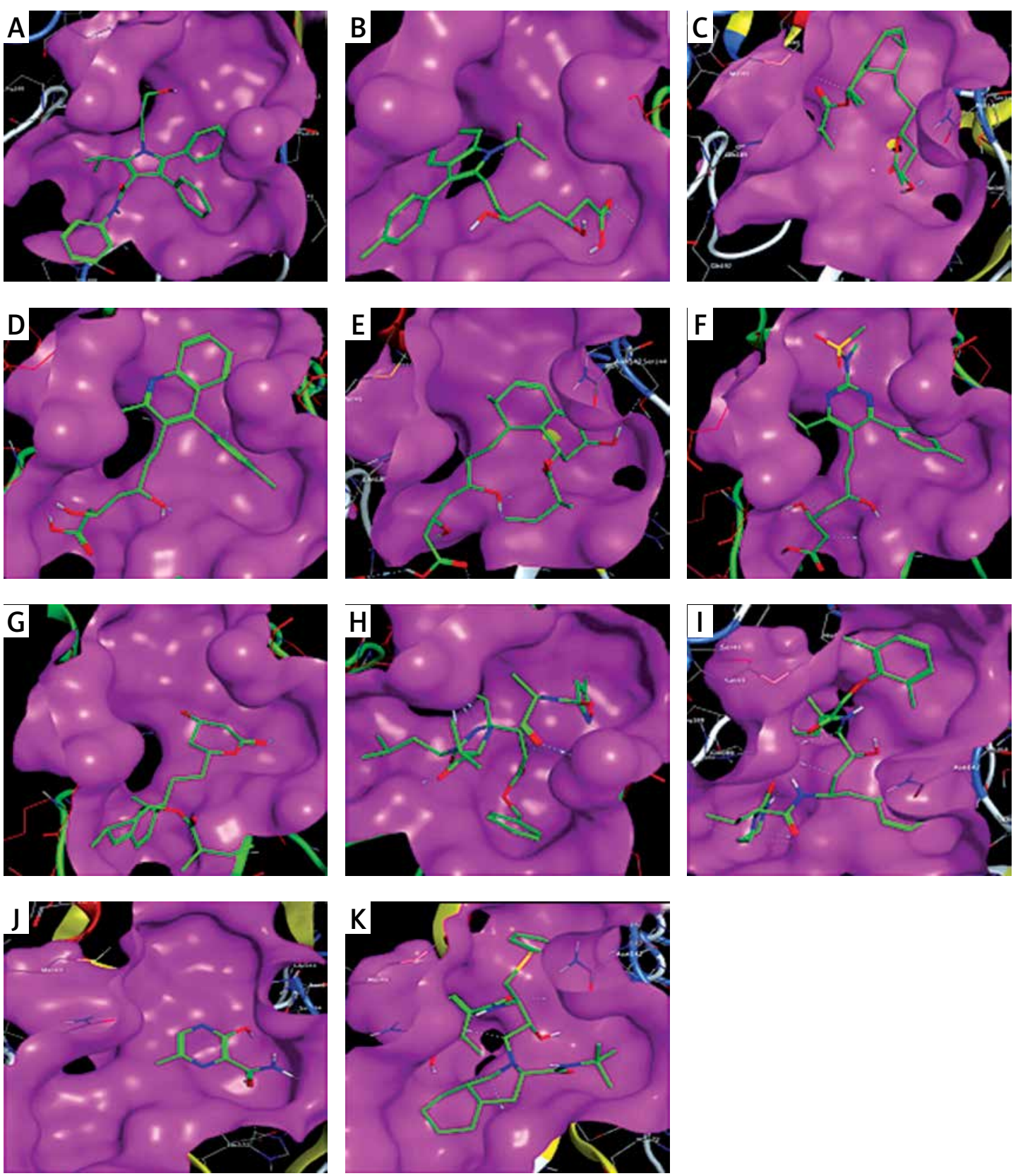

Figure 1. Docking analysis visualisation of 6 LU7 binding with atorvastatin (A), fluvastatin (B), lovastatin (C), pitavastatin (D), pravastatin (E), rosuvastatin (F), simvastatin (G), standard ligand (H), lopinavir (I), favipiravir (J), and nelfinavir $(\mathbf{K})$

\section{Molecular docking}

AutoDock/Vina, a computational docking program, was used for docking using COVID-19 Mpro and ligand data along with grid box characteristics in the configuration file. Results analysis was performed on the AutoDock Vina Result File using ADT. Finally, the top score poses were evaluated by using MOE to visualise protein-ligand interactions.

\section{Results}

We studied the affinity of seven statins (simvastatin, rosuvastatin, pravastatin, pitavastatin, lovastatin, fluvastatin, and atorvastatin), standard ligand, and protease inhibitors with the COVID-19 Mpro. Table I summarises the affinity $(\mathrm{Kcal} / \mathrm{mol})$, hydrogen bonds, and binding residues of these seven statins, standard ligand, and protease inhibitors with the COVID-19 Mpro, involved in COVID-19 infection. Statins had strong drug-like properties when docked along with known inhibitors against the Mpro. The ligands were sorted on the basis of binding strength and were compared with known inhibitors.

The binding energies from the interaction of 6LU7 docking site with standard ligand, favipira- 
vir, nelfinavir, lopinavir, simvastatin, rosuvastatin, pravastatin, pitavastatin, lovastatin, fluvastatin, and atorvastatin were: $-6.8,-5.8,-7.9,-7.9,-7.0$, $-7.7,-6.6,-8.2,-7.4,-7.7$, and $-6.8 \mathrm{kcal} / \mathrm{mol}$, respectively. The docking results based on the binding energy of pitavastatin, rosuvastatin, lovastatin, and fluvastatin suggest that these statins might be potential COVID-19 Mpro inhibitors. Docking visualisation of the binding of different statins, standard ligand, and protease inhibitors with 6 LU7 is shown in Figure 1.

\section{Discussion}

We performed an in silico molecular docking experiment on the interaction of statins with the main protease enzyme of SARS-CoV-2. Predisposition to acute cardiac complications related to existing atherosclerotic cardiovascular disease (ASCVD) may significantly increase the severity of COVID-19 as well as the mortality of these patients. This is particularly true for patients with high and very high risk for ASCVD, such as those with familial hypercholesterolaemia [21]. The main treatment for these patients is high-intensity statin therapy [22].

Statins have been suggested as useful drugs in COVID-19 patients mainly because of their capacity to decrease complications due to existing ASCVD (atheroma plaque stabilisation and reduction of CVD events and mortality) [23, 24] but also because of their anti-inflammatory and immunomodulatory effects, which might help to prevent acute lung injury in COVID-19 infection [25]. In addition, statins not only block virus replication upon antiviral activity but also reduce the harmful effects of inflammation on the host [26].

However, we investigated whether statins could directly interact with the main protease enzyme of SARS-CoV-2. We found that there is a strong effect of some statins, especially pitavastatin, with a binding energy (the more negative delta $G$ means stronger interaction), which is even greater than that of protease inhibitors. Docking studies may be of crucial value because until now no studies have investigated the effects of the chemical structure and inhibition of docking activity of statins on the main protease enzyme of SARS-CoV-2.

Because pitavastatin has already been shown to have a potent antiviral effect against Ebola virus [26], according to our results it could have a direct antiviral effect on SARS-CoV-2 as well. Based upon our results, rosuvastatin, lovastatin, and fluvastatin might also be useful in COVID-19 treatment.

The limitation of this study is in its in silico nature. Therefore, our results need to be confirmed by experimental studies, which might support our findings, as well as studies on patients with
COVID-19, for whom until today no specific treatment exists.

In conclusion, besides their indirect effects on COVID-19, such as decreasing complications due to existing ASCVD mostly because of their anti-inflammatory and immunomodulatory effects, our results suggest that statins can directly affect the virus particle. Therefore, their efficacy concerning COVID-19, especially for pitavastatin, warrants further investigation.

\section{Acknowledgments}

Željko Reiner and Mahdi Hatamipour equally contributed as the first author.

\section{Conflict of interest}

The authors declare of interest related to the topic of this paper.

\section{References}

1. Lee PI, Hsueh PR. Emerging threats from zoonotic coronaviruses-from SARS and MERS to 2019-nCoV. J Microbiol Immunol Infection 2020. doi: 10.1016/j.jmii. 2020.02.001

2. Rodríguez-Morales AJ, MacGregor K, Kanagarajah S, Patel D, Schlagenhauf P. Going global - Travel and the 2019 novel coronavirus. Travel Med Infect Dis 2020; 33 : 101578.

3. Katsiki N, Banach M, Mikhailidis D. Lipid-lowering therapy and renin-angiotensin-aldosterone system inhibitors in the era of the COVID-19 pandemic. Arch Med Sci 2020; 16: 485-9.

4. Nabavi S, Habtemariam S, Clementi E, et al. Lessons learned from SARS-CoV and MERS-CoV: FDA-approved Abelson tyrosine-protein kinase 2 inhibitors may help us combat SARS-CoV-2. Arch Med Sci 2020; 16 : 519-21.

5. Gbinigie K, Frie K. Should chloroquine and hydroxychloroquine be used to treat COVID-19? A rapid review. BJGP Open 2020. doi: 10.3399/bjgpopen20X101069.

6. Ye X, Luo Y, Xia S, et al. Clinical efficacy of lopinavir/ ritonavir in the treatment of Coronavirus disease 2019. Eur Rev Med Pharmacol Sci 2020; 24: 3390-6.

7. Dong L, Hu S, Gao J. Discovering drugs to treat coronavirus disease 2019 (COVID-19). Drug Discov Ther 2020; 14: 58-60.

8. Yende S, Milbrandt EB, Kellum JA, et al. Understanding the potential role of statins in pneumonia and sepsis. Crit Care Med 2011; 39: 1871-8.

9. Calis J, Lemson J. Severe sepsis and septic shock. N Engl J Med 2013; 369: 2062.

10. Chruściel P, Sahebkar A, Rembek-Wieliczko M, et al. Impact of statin therapy on plasma adiponectin concentrations: a systematic review and meta-analysis of 43 randomized controlled trial arms. Atherosclerosis 2016; 253: 194-208.

11. Parizadeh SMR, Azarpazhooh MR, Moohebati M, et al. Simvastatin therapy reduces prooxidant-antioxidant balance: results of a placebo-controlled cross-over trial. Lipids 2011; 46: 333-40.

12. Sahebkar A, Kotani K, Serban C, et al. Statin therapy reduces plasma endothelin-1 concentrations: a meta- 
analysis of 15 randomized controlled trials. Atherosclerosis 2015; 241: 433-42.

13. Sahebkar A, Serban C, Mikhailidis DP, et al. Association between statin use and plasma D-dimer levels: a systematic review and meta-analysis of randomised controlled trials. Thromb Haemost 2015; 114: 546-57.

14. Sahebkar A, Watts GF. New therapies targeting apoB metabolism for high-risk patients with inherited dyslipidaemias: what can the clinician expect? Cardiovasc Drugs Ther 2013; 27: 559-67.

15. Piechota M, Barylski M, Hannam S, Piechota-Urbanska M, Banach M. Rationale of statin therapy in septic patients. Curr Vasc Pharmacol 2013; 11: 795-800.

16. Shrivastava-Ranjan P, Flint M, Bergeron É, et al. Statins suppress Ebola virus infectivity by interfering with glycoprotein processing. MBio 2018; 9: e00660-18.

17. Khaerunnisa S, Kurniawan H, Awaluddin R, Suhartati S, Soetjipto S. Potential inhibitor of COVID-19 main protease (Mpro) from several medicinal plant compounds by molecular docking study. Preprints 2000, 2020030226. Doi: 10.20944/preprints202003.0226.v1. 2020: 1-14.

18. Liu X, Zhang B, Jin Z, Yang H, Rao Z. The crytal structure of 2019-NCoV main protease in complex with an inhibitor N3. RCSB Protein Data Bank 2020. DOI: 10.2210/ $\mathrm{pdb} 6 \mathrm{LU} 7 / \mathrm{pdb}$.

19. Xu Z, Peng C, Shi Y, et al. Nelfinavir was predicted to be a potential inhibitor of 2019-nCov main protease by an integrative approach combining homology modelling, molecular docking and binding free energy calculation. BioRxiv 2020. doi: https://doi.org/10.1101/ 2020.01.27.921627.

20. Jin Z, Du X, Xu Y, et al. Structure of Mpro from COVID-19 virus and discovery of its inhibitors. bioRxiv 2020. doi: https://doi.org/10.1101/2020.02.26.964882.

21. Vuorio A, Watts GF, Kovanen PT. Familial hypercholesterolemia and COVID-19: triggering of increased sustained cardiovascular risk. J Intern Med 2020. doi: 10.1111/ joim.13070.

22. Reiner Z. Management of patients with familial hypercholesterolaemia. Nat Rev Cardiol 2015; 12: 565-75.

23. Pirro M, Simental-Mendia LE, Bianconi V, Watts GF, Banach $M$, Sahebkar A. Effect of statin therapy on arterial wall inflammation based on 18F-FDG PET/CT: a systematic review and meta-analysis of interventional studies. J Clin Med 2019; 8(1). doi: 10.3390/jcm8010118.

24. Banach M, Serban C, Sahebkar A, et al. Impact of statin therapy on coronary plaque composition: a systematic review and meta-analysis of virtual histology intravascular ultrasound studies. BMC Med 2015; 13: 229.

25. Phadke M, Saunik S. COVID-19 treatment by repurposing drugs until the vaccine is in sight. Drug Dev Res 2020. doi: $10.1002 / d d r .21666$.

26. Fedson DS. A practical treatment for patients with Ebola virus disease. J Infect Dis 2015; 211: m661-2. 